internally filled with liquid ammonia, in molten phosphorus and lifting it so as to have it coated with a thin layer of crystallised white phosphorus. The capillary was then fixed in the axis of a Debye camera. The temperature may be estimated at $-35^{\circ} \mathrm{C}$.

The photograph obtained with the $K$ radiations of an iron anticathode shows 22 lines, which can all be arranged in the cubic system for a lattice constant of the elementary cell of $7 \cdot 17 \mathrm{~A}$. Supposing the cell as containing 4 molecules $\mathrm{P}_{4}$, the calculated density is $2 \cdot 23$, slightly greater than the data found in the literature at room temperature (values between 1.82 and $2 \cdot 0$ ).

The details of measurements and a discussion of the structure will be published elsewhere.

Several authors have deduced from cooling curves that at lower temperatures (less than c. $-70^{\circ} \mathrm{C}$.) another modification is formed, the form of which is still uncertain, since one of the authors describes it as hexagonal, and others as trimetric or monoclinic (P. W. Bridgmann : Jour. Am. Chem. Soc., 36, 1344 ; 1914. D. Vorländer, W. Selke, and S. Kreiss, Berichte, $58 \mathrm{~B}, 1802 ; 1925)$.

We repeated our observations, filling the capillary with liquid air. The photograph obtained in such a way (at c. $-170^{\circ}$ C.) shows a large number of lines which cannot be arranged in the cubic system.

The dimorphism of white phosphorus is thus confirmed. We cannot yet, on account of the complexity of the photographs, decide upon the symmetry of $\beta$-phosphorus, but we incline to tho opinion that it belongs to a system of rather low symmetry; at any rate we may conclude that it has a rather complex elementary cell. Investigations are still in progress. G. Natta. L. Passerint.

Laboratory of General Chemistry, Royal Polytechnic, Milan, Italy, April 4.

\section{A Diamagnetic Simple Salt of Nickel.}

ACCORDING to the accepted views on atomic structure, the atoms of nickel, palladium, and platinum should, in the fundamental state, show the same outer electronic structure, namely, $s^{2} d^{8}$, where $s$ denotes an electron with $l=0$ and $d$ an electron with $l=2$. Spectroscopic evidence seems to indicate that, in the case of palladium, the electronic distribution corresponding to the fundamental state is $d^{10}$. The magnetic properties of the compounds of these elements show great dissimilarity; for example, while $\mathrm{NiCl}_{2}$ is paramagnetic and has a magnetic moment of about 14-16 Weiss magnetons, both $\mathrm{PdCl}_{2}$ and $\mathrm{PtCl}_{2}$ are diamagnetic (Bose and Bhar, Zeit. f. Physik, Vol. 48, p. 716). This difference was explained by these authors on the assumption that in $\mathrm{NiCl}_{2}$ the two outer $s$ electrons of the nickel atom are transferred to the two $\mathrm{Cl}$ atoms giving rise to a polar molecule, while in the other two compounds the two chlorine atoms are in covalent bond with the two uncoupled $d$ electrons in the atoms respectively of palladium, or of platinum, thus neutralising the magnetic moments of these atoms. It has, however, been found that both $\mathrm{K}_{2} \mathrm{PtCl}_{4}$ and $\mathrm{K}_{2} \mathrm{Ni}(\mathrm{CN})_{4}$ are diamagnetic.

The magnetic similarity of these double salts of platinum and nickel made it worth while to investigate the properties of $\mathrm{Ni}(\mathrm{CN})_{2}$. Mr. Sushovan Dutt, working in this laboratory, has made the interesting discovery that while $\mathrm{Ni}(\mathrm{CN})_{2}, 7 \mathrm{H}_{2} \mathrm{O}$ is paramagnetic, and has a moment of the same order as the other divalent simple salts of nickel, this compound shows a progressive diminution of para- magnetism with dehydration, and it has been possible to obtain a salt with magnetic moment less than $\mathbf{2 . 5}$ Weiss magnetons. The salt is very hygroscopic, and in the process of transference from the desiccator to the sealed ampoule, into which it is introduced for magnetic measurements, it shows a perceptible change of colour. There seems to be no reason to doubt that on complete dehydration the compound would be found to be diamagnetic.

The observed facts can be accounted for on the assumption that, in the hydrated salt, one of the $s$ electrons of nickel is transferred to each of the (CN) groups, giving rise to polar bonds, while in the dehydrated state the two (CN) groups are attached by covalent bonds to the two uncoupled $d$ electrons of nickel. This is an interesting illustration of how the nature of the valency bond in a compound depends on its state of hydration, and of the suitability of the magnetic method for testing such bonds in compounds of paramagnetic elements. I am not aware of similar observations having been made before. Further investigations are proceeding.

University College of Science, D. M. Bose. Calcutta, Mar. 28.

\section{The Problem of Stellar Luminosity}

Though I have the very greatest respect for anything written by my friend and teacher Prof. Eddington, I cannot see that in his letter in NATURE of Mar. 29 he meets my arguments.

Prof. Eddington quotes a sentence of mine beginning "Similar considerations show . . . " and says that I leave this assertion unsupported. The considerations in question, which are of a simple physical character, are to be found in the two paragraphs immediately following the sentence quoted. These considerations have nothing to do with evolution or secular changes occurring in Nature. Prof. Eddington has not discussed these arguments, though they are at the very. basis of my line of thought.

My earlier paper in Monthly Notices R.A.S. (87, $708 ; 1927$ ), discusses a density-distribution in which the sinks of energy in the outer layers of the star exactly balance the source of energy in the inner portions, so that the luminosity of this density-distribution is zero.

The 'Humpty-Dumpty' argument shows that the uranium model possesses a stable configuration, which is all that I require. But my 'cooling' argument goes considerably further than this.

The problem discussed by Prof. Eddington in brief is: given the mass $M$ and the relative source-distribution, to find the luminosity $L$ in the steady state. The problem I discuss (suggested by the uranium model is : given the mass $M$ and the rate of generation $L$, to find the source-distribution in the steady state. I do this by considering a range of density. distributions, choosing those compatible with the arbitrarily assigned $L$ and $M$ and deducing the source distribution afterwards. The conditions that the outer layers shall simultaneously enclose mass $M$ and radiate to space at the rate $L$ impose a restriction on the possible density distributions, the restriction depending on the surface opacity. In this problem $L$ is a datum; it depends neither on mass, radius, nor opacity. The actual stars must be a subclass of the stars thus constructed. The observed mass-luminosity correlation must depend on the intrinsic nature of the energy-generating process; it is not accessible merely from steady-stato considerations.

Wadham College, Oxford, E. A. Milne.

No. 3158, VoL. 125] April 30 